\title{
A Review of Iris Recognition Algorithms
}

\author{
Richard Yew Fatt Ng Yong Haur Tay Kai Ming Mok \\ Computer Vision and Intelligent Systems (CVIS) Group \\ Universiti Tunku Abdul Rahman, Malaysia \\ richng01@yahoo.com, \{tayyh,mokkm\}@mail.utar.edu.my
}

\begin{abstract}
Iris recognition has become a popular research in recent years. Due to its reliability and nearly perfect recognition rates, iris recognition is used in high security areas. Among its applications are border control in airports and harbors, access control in laboratories and factories, identification for Automatic Teller Machines (ATMs) and restricted access to police evidence rooms. This paper provides a review of major iris recognition researches. There are three main stages in iris recognition system: image preprocessing, feature extraction and template matching. A literature review of the most prominent algorithms implemented in each stage is presented.
\end{abstract}

\section{Introduction}

Biometric identification is an emerging technology which gains more attention in recent years. It employs physiological or behavioral characteristics to identify an individual. The physiological characteristics are iris, fingerprint, face and hand geometry. Voice, signature and keystroke dynamics are classified as behavioral characteristics. Among these characteristics, iris has distinct phase information which spans about 249 degrees of freedom [2]. This advantage let iris recognition be the most accurate and reliable biometric identification.

The three main stages of an iris recognition system are image preprocessing, feature extraction and template matching.

The iris image needs to be preprocessed to obtain useful iris region. Image preprocessing is divided into three steps: iris localization, iris normalization and image enhancement. Iris localization detects the inner and outer boundaries of iris. Eyelids and eyelashes that may cover the iris region are detected and removed. Iris normalization converts iris image from Cartesian coordinates to Polar coordinates. The normalized iris image is a rectangle image with angular resolution and radial resolution. The iris image has low contrast and non-uniform illumination caused by the position of the light source. All these factors can be compensated by the image enhancement algorithms.

Feature extraction uses texture analysis method to extract features from the normalized iris image. The significant features of the iris are extracted for accurate identification purpose.

Template matching compares the user template with templates from the database using a matching metric. The matching metric will give a measure of similarity between two iris templates. It gives a range of values when comparing templates from the same iris, and another range of values when comparing templates from different irises. Finally, a decision of high confidence level is made to identify whether the user is an authentic or imposter.

The most prominent algorithms in each iris recognition stage would be discussed in next three sections. This paper discusses the pros and cons of algorithms implemented in each stage. Iris image capture stage will not be discussed in this paper. 


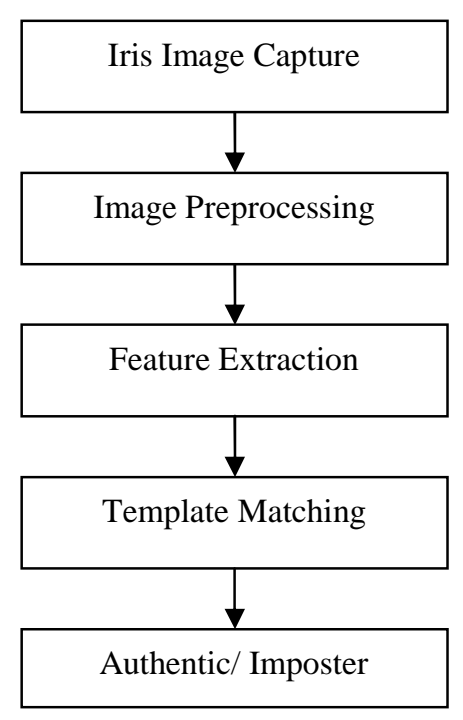

Figure 1. Stages of iris recognition algorithm

\section{Image preprocessing}

Iris image preprocessing is divided into three steps: iris localization, iris normalization and image enhancement.

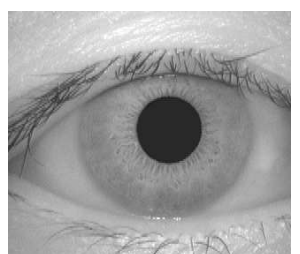

(a)

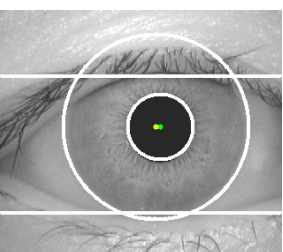

(b) (c)

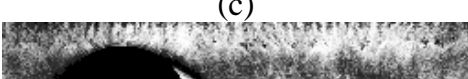

(d)

Figure 2. Iris image preprocessing (a) original iris image, (b) localized iris image, (c) normalized iris image, (d) enhanced iris image

\subsection{Iris localization}

Iris localization detects the inner and outer boundaries of the iris. Both the inner and outer iris boundaries can be approximately modeled as circles. The center of iris does not necessarily concentric with the center of pupil. Iris localization is important because correct iris region is needed to generate the templates for accurate matching. Five iris localization algorithms would be discussed in this section. They include Integro-differential operator, Hough transform, Discrete Circular Active Contour, Bisection method and Black hole search method. An example of localized iris image is shown in Fig. 2(b).

2.1.1 Integro-differential operator. Integrodifferential operator is used for locating the inner and outer boundaries of iris, as well as the upper and lower eyelids [1], [2].

The operator computes the partial derivative of the average intensity of circle points, with respect to increasing radius, $r$. After convolving the operator with Gaussian kernel, the maximum difference between inner and outer circle will define the center and radius of the iris boundary. For upper and lower eyelids detection, the path of contour integration is modified from circular to parabolic curve.

The operator is accurate because it searches over the image domain for the global maximum. It can compute faster because it uses the first derivative information.

2.1.2 Hough transform. Since the inner and outer boundaries of an iris can be modeled as circles, circular Hough transform is used to localize the iris [3]-[6]. Firstly, edge detector is applied to a gray scale iris image to generate the edge map. The edge map is obtained by calculating the first derivative of intensity values and thresholding the results. Gaussian filter is applied to smooth the image to select the proper scale of edge analysis.

The voting procedure is realized using Hough transform in order to search for the desired contour from the edge map. Assuming a circle with center coordinate $\left(x_{c}, y_{c}\right)$ and radius $r$, each edge point on the circle casts a vote in Hough space. The center coordinate and radius of the circle with maximum number of votes is defined as the contour of interest. For eyelids detection, the contour is defined using parabolic curve parameter instead of the circle parameter.

The disadvantage of Hough transform algorithm is that it is computationally intensive and therefore not suitable for real time applications. It requires a threshold value to generate the edge map. The selected threshold value may remove some critical edge points and result in false circle detection.

2.1.3 Discrete circular active contour. Active contour model has been used to localize iris [7], [8]. The contour is defined as a set of $n$ vertices connected as a simple closed curve. The movement of the contour is caused by internal and external forces acting on the vertices. The internal forces expand the contour into a 
perfect circle. The external forces push the contour inward.

The contour moves under the influence of the internal and external forces until it reaches equilibrium. The average radius and center of the contour obtained are the parameters of the iris boundary.

The discrete circular active contour search for the iris boundary is affected by the specular reflections from the cornea. Therefore, image preprocessing algorithm is required to remove the specular reflections.

2.1.4 Bisection method. In both [9] and [10], the bisection method is used to locate the center of the pupil. The center of the pupil is used as reference to detect the inner and outer boundaries of the iris. Firstly, edge detection is applied to the iris image to extract the edge information.

For every two points on the same edge component, bisection method is applied to draw the perpendicular lines to the center point. The center point with maximum number of line intersections is selected as the center of the pupil.

A virtual circle is drawn with reference to the center of the pupil and the radius is increased within a certain range. Two virtual circles with the largest number of edge points are chosen as the inner and outer boundaries of the iris.

Bisection method is affected by the non-uniform illuminations and glasses reflections. As a result, the iris inner boundary cannot be localized accurately. Similar to the discrete circular active contour method, image preprocessing algorithm is needed to remove the high intensity areas caused by illuminations and reflections.

2.1.5 Black hole search method. Black hole search method is used to compute the center and area of a pupil [11], [12]. Since the pupil is the darkest region in the image, this approach applies threshold segmentation method to find the region.

Firstly, a threshold is defined to identify the dark areas in the iris image. The dark areas are called as "black holes". The center of mass of these black holes is computed from the global image.

The area of pupil is the total number of those black holes within the region. The radius of the pupil can be calculated from the circle area formula.

Black hole search method is not suitable for iris image with dark iris. The dark iris area would be detected instead of the area of pupil.

\subsection{Eyelid and eyelash detection}

Eyelids and eyelashes may cover the iris region. Eyelids can be detected using texture segmentation and Daubechies wavelets method. The eyelashes detection algorithms consist of Gabor filter, variance of intensity and combination of both edge and region information.

2.2.1 Eyelid detection. Texture segmentation is adopted to detect upper and lower eyelids in [15]. The energy of high spectrum at each region is computed to segment the eyelashes. The region with high frequency is considered as the eyelashes area. The information of the pupil position is used in upper eyelashes segmentation. The upper eyelashes are fit with a parabolic arc. The parabolic arc shows the position of the upper eyelid. For lower eyelid detection, the histogram of the original image is used. The lower eyelid area is segmented to compute the edge points of the lower eyelid. The lower eyelid is fit with the edge points.

In [16], the Daubechies wavelets method is used to decompose the original image into four bands, $\mathrm{HH}$, HL, LH and LL. Canny edge detection is applied to the LH image. To minimize the influence of eyelashes, Canny edge detector is tuned to horizontal direction. The edge points that are close to each other are connected to detect the upper eyelid. The longest connected edge that fits with a parabolic arc is taken as the upper eyelid. To detect lower eyelid, the steps are repeated with lower iris boundary area.

2.2.2 Eyelash detection. Gabor filter and variance of intensity approaches are proposed for eyelash detection [17]. The eyelashes are categorized into separable eyelashes and multiple eyelashes. Separable eyelashes are detected using 1D Gabor filters. A low output value is obtained from the convolution of the separable eyelashes with the Gabor filter. For multiple eyelashes, the variance of intensity is very small. If the variance of intensity in a window is smaller than a threshold, the center of the window is considered as the eyelashes.

According to [18], both the edge and region information are used for noise detection. To speed up iris segmentation, the iris is roughly localized using filtering, edge detection and Hough transform. The localized iris is normalized to rectangular block. A bank of Gabor filters is used to extract the edge information based on phase congruency. The obtained edge information is combined with the region information to detect the eyelashes and pupil noise regions. 


\subsection{Iris normalization}

Iris may be captured in different size with varying imaging distance. Due to illumination variations, the radial size of the pupil may change accordingly. The resulting deformation of the iris texture will affect the performance of subsequent feature extraction and matching stages. Therefore, the iris region needs to be normalized to compensate for these variations. Fig. 2(c) shows the iris image after normalization.

2.3.1 Homogenous rubber sheet model. The homogeneous rubber sheet model algorithm remaps each pixel in the localized iris region from the Cartesian coordinates to polar coordinates [1], [2]. The non-concentric polar representation is normalized to a fixed size rectangular block.

The homogenous rubber sheet model accounts for pupil dilation, imaging distance and non-concentric pupil displacement. However, this algorithm does not compensate for the rotation variance.

\subsection{Image enhancement}

The normalized iris image has low contrast and nonuniform illumination caused by the light source position. The image needs to be enhanced to compensate for these factors.

Local histogram analysis is applied to the normalized iris image to reduce the effect of nonuniform illumination and obtain well-distributed texture image [19], [6].

Reflections regions are characterized by high intensity values close to 255 . A simple thresholding operation can be used to remove the reflection noise [18]. The iris image after enhancement is shown in Fig. 2(d).

\section{Feature extraction}

In this stage, texture analysis methods are used to extract the significant features from the normalized iris image. The extracted features will be encoded to generate a biometric template.

\subsection{Gabor filters}

2D Gabor filters are used to extract iris features in both [1] and [2]. Gabor filter's impulse response is defined by a harmonic function multiplied by a Gaussian function. It provides optimum localization in both spatial and frequency domains.
Each pattern is demodulated to extract its phase information using quadrature 2D Gabor wavelets. The phase information is quantized into four quadrants in the complex plane. Each quadrant is represented with two bits phase information. Therefore, each pixel in the normalized image is demodulated into two bits code in the template.

The phase information is extracted because it provides the significant information within the image [20]. It does not depend on extraneous factors, such as imaging contrast, illumination and camera gain [2].

A Log Gabor filter which is Gaussian on a logarithmic scale is proposed by [21]. It has strictly band pass filter to remove the DC components caused by background brightness [22].

\subsection{Wavelet transform}

Wavelet transform decomposes the iris region into components with different resolutions. The commonly used wavelets are Daubechies, Biorthogonal, Haar and Mexican Hat wavelet [9], [13], [14], [16], [23]-[25].

The advantage of wavelet transform over Fourier transform is that it has both space resolution and frequency resolution. The features are localized in both space and frequency domains with varying window sizes.

A bank of wavelet filters is applied to the normalized iris region. Each filter is tuned for each resolution with each wavelet defined by scaling functions. The output of the filters is encoded to generate a compact biometric template.

\subsection{Laplacian of Gaussian filter}

Laplacian of Gaussian filters are used to encode feature by decomposing the iris region [3], [26]. The filtered image is realized as a Laplacian pyramid. A cascade of Gaussian-like filters is applied to the image. The Laplacian pyramid is constructed with four levels to generate a compact biometric template.

This approach compresses the data to obtain significant data. The compressed data can be stored and processed effectively.

\subsection{Key local variations}

Key local variations are used to represent the characteristics of the iris [27]. The normalized iris image is decomposed into a set of 1D intensity signals. Dyadic wavelet transform is applied to each signal. Local extrema of the wavelet transform results correspond to sharp intensity variations of the original 
signal. The local maximum and minimum points are encoded into a feature vector. The feature vector is converted to a binary template with the same size as the normalized iris image.

\subsection{Hilbert transform}

Hilbert transform is used to extract significant information from iris texture [5]. Analytic image is constructed by the original image and its Hilbert transform. It can be used to analyze the iris texture. Emergent frequency and instantaneous phase is computed from the analytic image. Emergent frequency is formed by three different dominant frequencies of the analytic image. Instantaneous phase is the arctangent function of the real and imaginary parts of the analytic image. Feature vector is encoded by thresholding the emergent frequency and the instantaneous phase.

The advantage of this approach is computationally effective. The filtering is performed in the Fourier domain using pure real filters.

\subsection{Discrete cosine transform}

Iris is coded based on differences of discrete cosine transform (DCT) coefficients of rectangular patches [28]. The normalized image is divided into diagonal $8 \times 12$ patches. The average over width is windowed using a Hanning window to reduce the effects of noise. A similar Hanning window and DCT is applied to the patch along its length. The differences between the DCT coefficients of adjacent patches are obtained. A binary template is generated from the zero crossings of the differences between the DCT coefficients.

This coding method has low complexity and good interclass separation. It is superior to other approaches in terms of both speed and accuracy.

\section{Template matching}

The templates generated from the feature extraction stage need a corresponding matching metric. The matching metric compares the similarity between the templates. A threshold is set to differentiate between intra-class and inter-class comparisons.

\subsection{Hamming distance}

Hamming distance is defined as the fractional measure of dissimilarity between two binary templates [1], [2]. A value of zero would represent a perfect match. The two templates that are completely independent would give a Hamming distance near to 0.5. A threshold is set to decide the two templates are from the same person or different persons.

The fractional hamming distance is sum of the exclusive-OR between two templates over the total number of bits. Masking templates are used in the calculation to exclude the noise regions. Only those bits in the templates that correspond to ' 1 ' bit in the masking template will be used in the calculation.

The advantage of Hamming distance is fast matching speed because the templates are in binary format. The execution time for exclusive-OR comparison of two templates is approximately $10 \mu \mathrm{s}$ [2]. Hamming distance is suitable for comparisons of millions of template in large database.

\subsection{Weighted Euclidean distance}

Weighted Euclidean distance is used to compare two templates to identify an iris [19]. The templates are composed of integer values. Weighted Euclidean Distance is defined as a measure of similarity between two templates. It is calculated using Pythagorean Theorem to obtain the distance between two points.

An iris template is compared with all templates in the database. The two templates are matched if the Weighted Euclidean Distance is a minimum.

\subsection{Normalized correlation}

Normalized correlation between two representations is calculated for goodness of match [3], [26]. It is defined as the normalized similarity of corresponding points in the iris region. The correlations are performed over small blocks of pixels in four different spatial frequency bands.

Normalized correlation accounts for local variations in image intensity. However, normalized correlation method is not computationally effective because images are used for comparisons.

\subsection{Nearest feature line}

Nearest feature line is an efficient classification method in template matching stage [6]. Feature line passes through any two feature points of the same class. The feature line extracts more variations of the feature vector than the feature points [29].

The distance from a feature point to the feature line is calculated. The nearest feature line distance will be used in the classification stage. 


\section{Discussion}

An important factor which must be considered for iris recognition system is the effect of noise on its performance. There are four kinds of noises exist in a normalized iris image. They are eyelids, eyelashes, pupil and reflection noises. However, most of the researches do not account for all types of noises. The researches focus on iris localization and their noise removal algorithms are not effective. A complete solution for compensating all types of noises should be implemented to achieve higher accuracy rate.

Most researches use the publicly available iris database for experiments. The two most popular iris image databases are CASIA [30] and University of Bath database [31]. These databases have limited number of iris images and the images are captured under controlled condition. Larger database with diversified populations should be used for evaluating the iris recognition algorithms.

\section{Conclusion}

This paper provides a review of well known researches on iris recognition. The algorithms used in iris recognition are categorized into three stages: image preprocessing, feature extraction and template matching. The performance of each algorithm implemented in each stage is evaluated.

There are increasing demands on iris recognition due to its reliability and accuracy. If the iris recognition algorithms are optimized for low-cost dedicated hardware, it will be employed in various applications.

\section{References}

[1] J. Daugman (1993). "High Confidence Visual Recognition of Persons by a Test of Statistical Independence", IEEE Tans. Pattern Analysis and Machine Intelligence, vol.15, pp.1148-1161.

[2] J. Daugman (2004). "How iris recognition works", IEEE Trans. CSVT, vol. 14, no. 1, pp. $21-30$.

[3] R.P. Wildes (1997). "Iris Recognition: An Emerging Biometric Technology", Proceedings of the IEEE, vol.85, pp.1348-1363.

[4] W. Kong and D. Zhang (2001). "Accurate iris segmentation based on novel reflection and eyelash detection model", Proceedings of 2001 International Symposium on Intelligent Multimedia, Video and Speech Processing.
[5] C. Tisse, L. Martin, L. Torres, and M. Robert. (2002). "Person identification technique using human iris recognition”, International Conference on Vision Interface.

[6] L.Ma, Y. Wang, and T. Tan (2002). "Iris recognition using circular symmetric filters", International Conference on Pattern Recognition, vol.2, pp.414-417.

[7] N. Ritter (1999). "Location of the Pupil-Iris Border in Slit Lamp Images of the Cornea", Proceedings of the International Conference on Image Analysis and Processing.

[8] N. Ritter and J. Cooper (2003). "Locating the iris: A first step to registration and identification”, Proceedings of the $9^{\text {th }}$ IASTED International Conference on Signal and Image processing, pp. 507-512.

[9] S. Lim, K. Lee, O. Byeon, and T.Kim (2001). "Efficient Iris Recognition through Improvement of Feature Vector and Classifier", ETRI Journal, vol. 23, no.2, pp. 61-70.

[10] H. Sung, J. Lim, J. Park, and Y. Lee (2004). "Iris Recognition Using Collarette Boundary Localization", Proceedings of the $17^{\text {th }}$ International Conference on Pattern Recognition, vol. 4, pp. 857-860.

[11] C.C. Teo and H.T. Ewe (2005). "An Efficient OneDimensional Fractal Analysis for Iris Recognition”, Proceedings of the $13^{\text {th }}$ WSCG International Conference in Central Europe on Computer Graphics, Visualization and Computer Vision 2005, pp. 157-160.

[12] K. Grabowski, W. Sankowski, M. Zubert, and M. Napieralska (2006). "Reliable Iris Localization Method with Application to Iris Recognition in Near Infrared Light", MIXDES 2006.

[13] A. Poursaberi and B.N. Araabi (2007). "Iris Recognition for Partially Occluded Images: Methodology and Sensitivity Analysis", EURASIP Journal on Advances in Signal Processing, vol 2007.

[14] A. Poursaberi and B.N. Araabi (2005). "A Novel Iris Recognition System Using Morphological Edge Detector and Wavelet Phase Features", GVIP (05), No. V6, pp. 9-15.

[15] J. Cui, Y. Wang, T. Tan, L. Ma, and Z. Sun (2004). “A Fast and Robust Iris Localization Method Based on Texture Segmentation", SPIE Defense and Security Symposium, vol. 5404, pp. 401-408.

[16] Y. Chen, S. Dass, and A. Jain (2006). "Localized Iris Image Quality Using 2D Wavelets", Proceedings of International Conference on Biometrics.

[17] W. Kong and D. Zhang (2001). "Accurate iris segmentation based on novel reflection and eyelash detection model", Proceedings of 2001 International Symposium on Intelligent Multimedia, Video and Speech Processing. 
[18] J. Huang, Y. Wang, T. Tan, and J. Cui (2004). “A New Iris Segmentation Method for Recognition", Proceedings of the $17^{\text {th }}$ International Conference on Pattern Recognition.

[19] Y. Zhu, T. Tan, and Y. Wang (2000). "Biometric Personal Identification Based on Iris Patterns", Proceedings of the $15^{\text {th }}$ International Conference on Pattern Recognition, vol. 2, pp. 2801-2804.

[20] A. Oppenheim and J. Lim (1981). "The importance of phase in signals", Proceedings of the IEEE 69, pp. 529-541.

[21] D. Field (1987). "Relations between the statistics of natural images and the response properties of cortical cells", Journal of the Optical Society of America.

[22] P. Yao, J. Li, X. Ye, Z. Zhuang, and B. Li (2006). "Iris Recognition Algorithm Using Modified Log-Gabor Filters", Proceedings of the $18^{\text {th }}$ International Conference on Pattern Recognition.

[23] E. Rydgren, T. Ea, F. Amiel, F. Rossant, and A. Amara (2004). "Iris Feature Extraction Using Wavelet Packets", IEEE International Conference on Image Processing. Vol. 2, pp. 861-864.

[24] W. Boles and B. Boashash (1998). "A human identification technique using images of the iris and wavelet transform", IEEE Transactions on Signal Processing, vol. 46, no. 4.

[25] C. Sanchez-Avila, R. Sanchez-Reillo, and D. De MartinRoche (2002). "Iris-Based Biometric Recognition Using Dyadic Wavelet Transform", IEEE AESS System Magazines, vol. 17 , no. 10 , pp. 3-6.

[26] R. Wildes, J. Asmuth, G. Green, S. Hsu, R. Kolczynski, J. Matey, and S. McBride (1994). "A System for Automated Iris Recognition", Proceedings of the IEEE Workshop on Applications of Computer Vision, pp. 121-128.

[27] L. Ma, T. Tan, Y. Wang, and D. Zhang (2004). "Efficient Iris Recognition by Characterizing Key Local Variations", IEEE Trans. Image Processing, vol 13, no.6, pp. 739-750.

[28] D. M. Monro, S. Rakshit, and D. Zhang (2007). "DCTBased Iris Recognition", IEEE Transactions on Pattern Analysis and Machine Intelligence, vol. 29, no. 4.

[29] S.Z. Li and J. Lu (1999). "Face Recognition Using the Nearest Feature Line Method", IEEE Transactions on Neural Network, 10(2), pp. 439-443.

[30] "CASIA Iris Image Database," http://www.sinobiometrics.com/Databases.htm, 2007.

[31] "University of Bath Iris Image Database," http://www.bath.ac.uk/elec-

eng/research/sipg/irisweb/database.htm, 2007. 